\title{
LINEAR EQUATIONS WITH UNSYMMETRIC SYSTEMS OF
}

\section{COEFFICIENTS*}

BY

\section{A. J. PELL}

In two previous papers $\nmid$ we have considered cases of integral equations

$$
\lambda \phi(s)=\int_{a}^{b} K(s, t) \phi(t) d t,
$$

with unsymmetric kernel $K(s, t)$, which have real characteristic functions, in terms of which $\int_{a}^{b} \int_{a}^{b} K(s, t) f(s) g(t) d s d t$ may be expanded. In this paper we consider the corresponding theory for linear equations in infinitely many unknowns, which includes the theory of integral equations as a special case. The method is the same as that used by us for integral equations, reduction, by means of a biorthogonal system, to a system of linear equations with a limited symmetric matrix of coefficients. We find in Section 4 that, for a limited matrix $A$, a necessary and sufficient condition for the existence of real characteristic forms of the equations

$$
\lambda l_{i}=\sum_{k} a_{i k} l_{k}, \quad \int_{\Delta} \lambda d p_{i}=\sum_{k} a_{i k} \Delta p_{k},
$$

which form a complete system with limited linear forms $F_{i}(x)$ such that $A F_{i}(x)=0$, or in terms of which $A(x, y)$ may be expanded, is the existence of a positive definite, symmetric, and limited matrix $T$ such that $A T$ is symmetric. The condition obtained by us on the kernel $K(s, t)$ for integral equations, is that there exist a functional transformation $¥ T$ such that $T_{s} K(s, t)$ is symmetric. This functional transformation differs only slightly from the one, which by the Riesz-Fischer theorem corresponds to the matrix $T$, the additional restriction being that it transform every continuous function into a continuous function.

* Presented to the Society, September, 1910.

$\dagger$ Bulletin of the American Mathematical Society, July, 1910, pp. 513-515, and these Transactions, vol. 12, pp. 165-180.

$\ddagger \mathrm{J}$. Marty obtained necessary and sufficient conditions for the problem in integral equations, expressed in terms of a special functional transformation $T(f)=\int_{a}^{b} k(s, t) f(t) d t$, Comptes Rendus, April, 1910, and June, 1910. 
Section 3 deals with properties of biorthogonal systems of linear forms and linear differential forms.

\section{Notation}

We confine our attention to real constants and real functions of real variables. If the range of a variable is that of a sequence, finite or infinite, it is indicated by the subscripts $i, j, k, l, m, \alpha$, and $\beta$. Similarly, constants and functions with these subscripts denote sequences, finite or infinite, of the constants and functions.

The coefficients in the linear form $F(x)$ of the variables $\left\{x_{i}\right\}$ are denoted by the corresponding small letters $f_{i}$, thus

$$
F(x)=\sum_{i} f_{i} x_{i}
$$

The increment of a continuous function $f(\lambda)$ for an interval $\Delta=\left(\lambda_{1}, \lambda_{2}\right)$ is denoted by $\Delta f(\lambda)=f\left(\lambda_{2}\right)-f\left(\lambda_{1}\right)$. If $\Delta_{1}$ and $\Delta_{2}$ are two intervals of $\lambda$ then $\Delta_{12} f(\lambda)$ is the increment of $f(\lambda)$ in the interval common to $\Delta_{1}$ and $\Delta_{2}$ if they overlap, and is zero if they do not overlap.

Matrices are denoted by capital letters. The elements of a matrix $A$ are denoted by the corresponding small letters $a_{i k}$ and we write $A=\left(a_{i k}\right)$. The elements of the matrix $A^{\prime}$ are $a_{i k}^{\prime}=a_{k i}$. The corresponding quadratic and bilinear forms in the variables $\left\{x_{i}\right\}$ and $\left\{y_{i}\right\}$ are denoted by $A(x, x)$ and $A(x, y)$ respectively.

The unit matrix is denoted by $E$

$$
e_{i k}= \begin{cases}1 & i=k \\ 0 & i \neq k\end{cases}
$$

If $F(x)$ and $G(x)$ are two linear forms, the symbol $(F, G)$ denotes the following operation

$$
(F, G)=\sum_{i} f_{i} g_{i}
$$

For the matrices $A$ and $B$ we write

$$
A B=\left(\sum_{j} a_{i j} b_{j k}\right) .
$$

The transformation of a linear form $F(x)$ by a matrix $A$ is expressed by

$$
A F(x)=\sum_{i}\left(\sum_{j} a_{i j} f_{j}\right) x_{i}
$$

2. Definitions and general properties of Linear forms aNd Matrices

Limited linear forms. If for a sequence $\left\{c_{i}\right\}$ the sum $\sum_{i} c_{i}^{2}$ converges, it is called the norm of $\left\{c_{i}\right\}$. and the sequence is of finite norm. A linear 
form $F(x)$ is limited, if there exists a quantity $M$ such that for every sequence $\left\{x_{i}\right\}$ of finite norm, and for every $n$,

$$
\left|\sum_{i=1}^{n} f_{i} x_{i}\right| \leqq M \sqrt{\sum_{i=1}^{n} x_{i}^{2}}
$$

(A) A linear form $F(x)$ is limited,* when and only when the coefficients $\left\{f_{i}\right\}$ are of finite norm, and for two limited linear forms $F(x)$ and $G(x)$, $(F, G)$ converges.

If $(F, G)=0$, the two linear forms $F(x)$ and $G(x)$ are orthogonal.

Limited matrices. A matrix $A$ and the corresponding quadratic and bilinear forms are limited, if there exists a positive quantity $M$ such that for all sequences $\left\{x_{i}\right\}$ and $\left\{y_{i}\right\}$ of finite norm, and for every $n$,

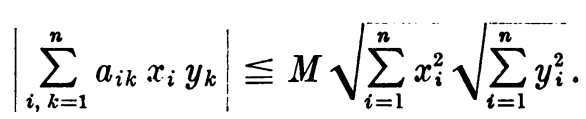

(B) If for every limited linear form $F(x)$, the linear form $A F(x)$ is also limited, the matrix $A$ is limited. $\dagger$

(C) If $A$ is a limited matrix, $\ddagger$ and the sequences $\left\{x_{i}\right\}$ and $\left\{y_{i}\right\}$ are of finite norm,

$$
\sum_{i}\left(\sum_{k} a_{i k} x_{i} y_{k}\right)=\sum_{k}\left(\sum_{i} a_{i k} x_{i} y_{k}\right)
$$

(D) If $A$ and $B$ are limited matrices, $\$$ the matrix $A B$ exists and is limited. A system of linear forms $\left\{L_{i}(x)\right\}$ forms an orthogonal system if $\left(L_{i}, L_{k}\right)=e_{i k}$.

(E) The matrix $L$ corresponding to an orthogonal $\|$ system of limited linear forms $L_{i}(x)$ is limited.

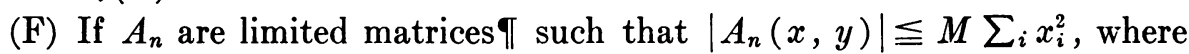
$M$ is independent of $n$, and if $\lim a_{i k}^{(n)}=a_{i k}$, then $A$ is a limited matrix and $|A(x, x)| \leqq M \sum_{i} x_{i}^{2}$.

A matrix $B$ is the front reciprocal of the matrix $A$ if $A B=E$ and the rear reciprocal of $A$ if $B A=E$.

* Hilbert, Grundzüge einer allgemeinen Theorie der Integralgleichungen, p. 126.

$\dagger$ Hellinger and Toeplitz, Theoric der unendlichen Matrizen, $\S 10, \mathrm{M}$ a t $\mathrm{h}$ e $\mathrm{m}$ a $\mathrm{t}$ is $\mathrm{c} \mathrm{h}$ e A n n a l e n, vol. 69, 1910. See also E. Schmidt, Ueber die Auflösung linearer Gleichungen mit unendlichvielen Unbekannten, Rendiconti del Circolo Matematico di P a l e r m o, t. 25,1908, p. 54 .

$\ddagger$ Hilbert, l. c., p. 120 .

§ Hilbert, l. c., pp. 128-129.

II Hilbert, l. c., p. 130.

I Riesz, Les systèmes d'équalions linéaires à une infinité d'inconnues, p. 106. 
Stieltjes integral.* Let $f(x)$ be a continuous function of limited variation in the interval $(a, b)$, and let the interval be divided into subintervals $\Delta_{i}=\left(\lambda_{i}, \lambda_{i+1}\right)$ by the points $\lambda_{0}=a, \lambda_{1}, \cdots, \lambda_{n-1}, \lambda_{n}=b$, in such a way that $\Delta_{i}$ approaches zero as $n$ increases, and let $u_{i}$ be any value of a continuous function $u(\lambda)$ in $\Delta_{i}$, then the sum

$$
\sum_{i=1}^{n} u_{i} \Delta_{i} f(\lambda)
$$

has a limit, and it is denoted by the Stieltjes integral

$$
\int_{a}^{b} u(\lambda) d f(\lambda)
$$

and this integral is a continuous function of the upper limit of integration.

Hellinger integrals. Let $f(x)$ be a continuous function, and $f_{0}(\lambda)$ be a continuous, monotonic non-decreasing function of $\lambda$ in $(a, b)$, and let $f(\lambda)$ be constant in every interval of $(a, b)$ in which $f_{0}(\lambda)$ is constant. Divide $(a, b)$ into $n$ subintervals $\Delta_{i}=\left(\lambda_{i}, \lambda_{i+1}\right)$ by the points, $\lambda_{0}=a, \lambda_{1}, \cdots$, $\lambda_{n-1}, \lambda_{n}=b$, in such a way that $\Delta_{i}$ approaches zero, then the sum

$$
\sum_{i=1}^{n} \frac{\left(\Delta_{i} f(\lambda)\right)^{2}}{\Delta_{i} f_{0}(\lambda)}
$$

has a limit, and it is denoted by the Hellinger integral

$$
\int_{a}^{b} \frac{(d f(\lambda))^{2}}{d f_{0}(\lambda)}
$$

A sufficient condition that the Hellinger integral exist is that $(\Delta f)^{2} \leqq \Delta h \Delta f_{0}$, where $h(\lambda)$ is a continuous, monotonic non-decreasing function in $\lambda$. If the integral with the upper limit $\lambda$ is called $h(\lambda)$, then $h(\lambda)$ is a continuous, monotonic function, and

$$
(\Delta f)^{2} \leqq \Delta h \Delta f_{0},
$$

and hence $f(\lambda)$ is a function of limited variation.

The system of functions $\left\{f^{(a)}\right\}$ is integrable $H\left(f_{0}^{(a)}\right)$ on an interval $(a, b)$, if $f^{(a)}$ are continuous functions, $f_{0}^{(a)}$ are continuous, monotonic non-decreasing functions of $\lambda$ in the interval $(a, b)$, if the integrals $\int_{a}^{b}\left(d f^{(a)}\right)^{2} / d f_{0}^{(a)}$ exist, $\dagger$ and the sum

converges.

$$
\sum_{a} \int_{a}^{b} \frac{\left(d f^{(a)}\right)^{2}}{d f_{0}^{(a)}}
$$

* The statements concerning Stieltjes and Hellinger integrals are results taken from Hellinger, Neue Begründung der Theorie quadratischer Formen von unendlichvielen Veränderlichen, J o u rnal f ür M thematik, vol. 136, pp. 234-242, or are easily derived from them.

$\dagger$ The argument in the integral is omitted whenever there is no ambiguity. 
If $f(\lambda)$ and $f_{1}(\lambda)$ are both integrable $H\left(f_{0}\right)$ on the interval $(a, b)$, and $u_{i}$ is the value of a continuous function $u(\lambda)$ at some point in $\Delta_{i}$, the sum

$$
\sum_{i=1}^{n} u_{i} \frac{\Delta_{i} f \Delta_{i} f_{1}}{\Delta_{i} f_{0}}
$$

has a limit, and it is denoted by

$$
\int_{a}^{b} u \frac{d f d f_{1}}{d f_{0}}
$$

Linear differential forms. If $f_{i}(\lambda)$ are continuous functions in the interval $(a, b)$, the system of linear form $\Delta F(\lambda ; x)=\sum_{i} \Delta f_{i}(\lambda) x_{i}$ for all possible subintervals $\Delta$ of $(a, b)$, forms a system of linear differential forms, for which we use the symbol $d F(\lambda ; x)=\sum_{i} d f_{i}(\lambda) x_{i}$, and denote the matrix of coefficients $\Delta f_{i}(\lambda)$ by $d F$.

The linear differential forms $d F(\lambda ; x)$ are limited, if the functions are continuous in the interval $(a, b)$, and if the norm $f_{0}(\lambda)$ is continuous in $(a, b)$.

Given a system of limited linear differential forms $\left\{d F^{(a)}(\lambda ; x)\right\}$ and an interval $(a, b) ;\left\{d F^{(a)}\right\}$ is limited with respect to $\left\{f_{0}^{(a)}\right\}$, if $f_{0}^{(a)}$ are continuous, monotonic non-decreasing functions in $(a, b)$, if $\left\{d F^{(a)}(\lambda ; x)\right\}$ is integrable $H\left(f_{v}^{(a)}\right)$, and if there exists a quantity $M$ such that for every sequence $\left\{x_{i}\right\}$ of finite norm

$$
\sum_{a} \int_{a}^{b} \frac{\left(d F^{(a)}(\lambda ; x)\right)^{2}}{d f_{0}^{(a)}(\lambda)} \leqq M \sum_{i} x_{i}^{2} .
$$

(G) If $\left\{f^{(a)}\right\}$ is integrable $H\left(f_{0}^{(a)}\right)$ and if $\left\{d F^{(a)}\right\}$ is limited with respect to $\left\{f_{0}^{(a)}\right\}$ for an interval $(a, b)$, then

$$
\sum_{a} \int_{a}^{b} \frac{d F^{(a)}(\lambda ; x) d f^{(a)}(\lambda)}{d f_{0}^{(a)}(\lambda)}=\sum_{i} x_{i}\left(\sum_{a} \int_{a}^{b} \frac{d f_{i}^{(a)} d f^{(a)}}{d f_{0}^{(a)}}\right),
$$

and is a limited linear form. If $\left\{d G^{(a)}(\lambda ; x)\right\}$ is also a system of differential forms, limited with respect to $\left\{f_{0}^{(a)}\right\}$, and $u(\lambda)$ is a continuous function,

$$
\sum_{a} \int_{a}^{b} u(\lambda) \frac{d F^{(a)}(\lambda ; x) d G^{(a)}(\lambda ; y)}{d f_{0}^{(a)}(\lambda)}=\sum_{i, k} x_{i} y_{k}\left(\int_{a}^{b} u \cdot \frac{d f_{i}^{(a)} d f_{k}^{(a)}}{d f_{0}^{(a)}}\right),
$$

and is a limited bilinear form.

A system consisting* of limited linear forms $\left\{L_{i}(x)\right\}$, and limited linear differential forms $\left\{d P^{(a)}(\lambda ; x)\right\}$, for an interval $(a, b)$, forms a normalized orthogonal system with basis functions $\left\{p_{0}^{(a)}(\lambda)\right\}$, if

$$
\left(L_{i}, L_{k}\right)=e_{i k}
$$

* It is to be understood throughout this paper, that in a system consisting of linear forms $\left\{L_{i}(x)\right\}$, and linear differential forms $\left\{d P^{(a)}(\lambda ; x)\right\}$, either ali the $L_{i}(x)$ or all the $d P(\alpha)(\lambda ; x)$ may be zero. 
if for any two intervals $\Delta_{1}$ and $\Delta_{2}$ of $(a, b)$

$$
\left(\Delta_{1} P^{(a)}, \Delta_{2} P^{(\beta)}\right)=\left\{\begin{array}{cc}
\Delta_{12} p_{0}^{(a)}(\lambda) & \alpha=\beta, \\
0 & \alpha \neq \beta
\end{array}\right.
$$

and if for any interval $\Delta$ of $(a, b)$

$$
\left(L_{i}, \Delta P^{(a)}\right)=0 .
$$

Since the relation (3) involves only the increments of $p_{i}^{(a)}(\lambda)$ we assume for an orthogonal system that $p_{i}^{(a)}\left(\lambda_{0}\right)=0$ for some point $\lambda_{0}$ of $(a, b)$. It follows that

$$
p_{0}^{(a)}(\lambda)=\left\{\begin{aligned}
\sum_{i}\left(p_{i}^{(a)}(\lambda)^{2}\right) & \lambda-\lambda_{0}>0 \\
-\sum_{i}\left(p_{i}^{(a)}(\lambda)^{2}\right) & \lambda-\lambda_{0}<0
\end{aligned}\right.
$$

and the $p_{0}^{(a)}(\lambda)$ are continuous, monotonic non-decreasing functions of $\lambda$. The orthogonal relation (3) is equivalent* to

$$
\sum_{i} \int_{a}^{b} \frac{d f^{(\alpha)} d p_{i}^{(\alpha)}}{d p_{0}^{(\alpha)}} \int_{a}^{b} \frac{d f_{1}^{(\beta)} d p_{i}^{(\beta)}}{d p_{0}^{(\beta)}}=\left\{\begin{array}{cc}
\int_{a}^{b} \frac{d f^{(\alpha)} d f_{1}^{(\alpha)}}{d p_{0}^{(\alpha)}} & \alpha=\beta \\
0 & \alpha \neq \beta
\end{array}\right.
$$

where $\left\{f^{(a)}\right\}$ and $\left\{f_{1}^{(a)}\right\}$ are any functions integrable $H\left(p_{0}^{(a)}\right)$.

(H) An orthogonal $\dagger$ system of linear differential forms $\left\{d P^{(a)}(\lambda ; x)\right\}$ with basis functions $\left\{p_{0}^{(a)}\right\}$ for an interval $(a, b)$, is limited with respect to $\left\{p_{0}^{(a)}\right\}$.

In conformity with the notation for the composition of two matrices, both of whose arguments have the range of a sequence, we adopt the following notation, when there is no ambiguity about the basis functions $\left\{f_{0}^{(a)}\right\}$,

$$
\sum_{a} \int_{a}^{b} \frac{d f_{t}^{(a)} d g_{k}^{(a)}}{d f_{0}^{(a)}}=F^{\prime} G_{a}^{b}=F^{\prime} G
$$

according as there is, or is not any ambiguity about the limits of integration. The product of the matrix above with a matrix $A=\left(a_{i k}\right)$ is indicated by

and

$$
\left(\sum_{j} a_{i j}\left(\sum_{a} \int_{a}^{b} \frac{d f_{j}^{(a)} d g_{k}^{(a)}}{d f_{0}^{(a)}}\right)\right)=A F^{\prime} G_{a}^{b}=A F^{\prime} G,
$$

$$
\left(\sum_{j}\left(\sum_{a} \int_{a}^{b} \frac{d f_{i}^{(a)} d g_{j}^{(a)}}{d f_{0}^{(a)}}\right) a_{j k}\right)=F^{\prime} G_{a}^{b} A=F^{\prime} G A
$$

A system of linear forms is complete if there exists no limited linear form orthogonal to all the forms of the system.

* Hellinger, 1. c., p. 250.

† Hellinger, l. c., pp. 248 and 251. 
If the orthogonal system consisting of the linear forms $\left\{L_{i}(x)\right\}$, and the linear differential forms $\left\{d P^{(a)}(\lambda, x)\right\}$ with the basis functions $\left\{p_{0}^{(a)}(\lambda)\right\}$ for the interval $(a, b)$, is complete, then

$$
L^{\prime} L+P^{\prime} P=E \text {. }
$$

Linear equations with symmetric systems of coefficients.

A characteristic linear form of the system of equations

$$
\lambda l_{i}=\sum_{k} a_{i k} l_{k}
$$

where the matrix $A$ of coefficients is limited, is a limited linear form $L(x)$ not identically zero, whose coefficients $l_{i}$ for some value of $\lambda$, called characteristic number, satisfy the equations (5).

The characteristic numbers form the point spectrum.

A system of characteristic linear differential forms for an interval $(a, b)$ of the equations

$$
\int_{\Delta} \lambda d p_{i}(\lambda)=\sum_{k} a_{i k} \Delta p_{k}(\lambda)
$$

where the matrix $A$ of coefficients is limited, is a system of linear differential forms $\{d P(\lambda ; x)\}$ not identically zero, limited with respect to a continuous, monotonic non-decreasing function $p_{0}(\lambda)$, and such that the equations (6) are satisfied for every subinterval $\Delta$ of $(a, b)$.

The intervals of $\lambda$, for which characteristic linear differential forms exist, form the continuous spectrum.

(I) If $A$ is a limited symmetric* matrix, there exists a spectrum associated with it, which lies within a finite interval of the $\lambda$-axis. The characteristic linear forms $\left\{L_{i}(x)\right\}$, corresponding to the characteristic numbers $\left\{\lambda_{i}\right\}$, and the characteristic linear differential forms $\left\{d P^{(a)}(\lambda ; x)\right\}$ corresponding to the continuous spectrum $(a, b)$, form an orthogonal $\dagger$ system for $(a, b)$, and the basis functions are given by

$$
p_{0}^{(a)}(\lambda)=\left\{\begin{aligned}
\sum_{i}\left(p_{i}^{(a)}(\lambda)^{2}\right) & \lambda>0, \\
-\sum_{i}\left(p_{i}^{(a)}(\lambda)^{2}\right) & \lambda<0
\end{aligned}\right.
$$

under the assumption that $p_{i}^{(a)}(0)=0$. For sequences $\left\{x_{i}\right\}$ and $\left\{y_{i}\right\}$ of finite norm,

$$
\text { (7) } \quad A(x, y)=\sum_{i} \lambda_{i} L_{i}(x) L_{i}(y)+\sum_{a} \int_{a}^{b} \lambda \cdot \frac{d P^{(a)}(\lambda ; x) d P^{(a)}(\lambda ; y)}{d p_{0}^{(a)}(\lambda)}
$$

* Hilbert, 1. c., Kap. 11, and Hellinger, 1. c., pp. 210-271.

$\dagger$ The argument given by Hellinger, l. c., pp. 244-246, for the orthogonality covers only the case in which the spectrum is positive; it can however be extended to cover the general case. 
A limited symmetric matrix $A$ is positive if for every sequence of finite norm

$$
\sum_{i, k} a_{i k} x_{i} x_{k} \geqq 0,
$$

the equality sign holding only for $\sum_{k} a_{i k} x_{k}=0, i=1,2, \cdots$; and positive definite, if the equality sign holds only for $x_{i}=0, i=1,2, \cdots$.

(J) For a positive symmetric limited matrix of coefficients the spectrum contains no points to the left of the origin. if

whenever

A function $F(x)$ of the variables $\left\{x_{i}\right\}$ of finite norm is completely continuous

$$
\lim _{n=\infty} F\left(x_{i}+\epsilon_{i}^{(n)}\right)=F\left(x_{i}\right),
$$

$$
\lim _{n=\infty} \epsilon_{i}^{(n)}=0 \quad(i=1,2, \cdots) .
$$

(K) For a completely continuous* quadratic form $A(x, x)$, there exist no characteristic linear differential forms of the equations (6), and if for a value of $\lambda$ there exists a characteristic linear form of (5), then there exists a characteristic linear form for the same value of $\lambda$ of the adjoint system of equations

$$
\lambda l_{i}=\sum_{k} a_{k i} l_{k} .
$$

(L) A quadratic form $A(x, x)$ is completely continuous $\dagger$ if $\sum_{i, k} a_{i k}^{2}$ converges; also if $A=B C$, where $B(x, x)$ is limited and $C(x, x)$ is completely continuous.

\section{Biorthogonal Systems}

Two finite or infinite systems of limited linear forms $\left\{U_{i}(x)\right\}$ and $\left\{V_{i}(x)\right\}$ form a biorthogonal system if

$$
\left(U_{i}, V_{k}\right)=e_{i k} .
$$

This condition could be written

$$
U V^{\prime}=E,
$$

which expresses that $U$ is the rear reciprocal of $V^{\prime}$. In a biorthogonal system of limited linear forms $\left\{U_{i}(x)\right\}$ and $\left\{V_{i}(x)\right\}$ each system is linearly independent, and the rows of the matrices $U$ and $V$ are of finite norm (A $\S 2$ ), although the columns may not be. The matrices may both be limited, or both unlimited, or one may be limited and the other unlimited.

Theorem I. If $T$ is a limited, positive, symmetric matrix, not identically zero, there exists a biorthogonal system, finite or infinite, of limited linear forms $\left\{U_{i}(x)\right\}$ and $\left\{V_{i}(x)\right\}$, for which the matrix $V$ is limited, and

* Hilbert, l. c., pp. 165-170.

$$
V_{i}(x)=T U_{i}(x), \quad T=V V^{\prime} .
$$

$\dagger$ Hilbert, l. c., pp. 150 and 176. 
Let $\left\{f_{i}(x)\right\}$ be a system of linearly independent limited linear forms, such that $T F_{i} \neq 0$ and either the system is complete or forms a complete system together with limited linear forms $\left\{\bar{F}_{i}(x)\right\}$, such that $\overline{T F}_{i}(x)=0$ and which form a linearly independent system together with the $\left\{F_{i}(x)\right\}$. For example let $F_{i}(x)=T_{i}(x)$, omitting those $T_{i}(x)$ which are linearly dependent on a finite number of the preceding. Then the system

$$
G_{i}(x)=T F_{i}(x)
$$

is linearly independent. The biorthogonal system is constructed as follows

where

$$
\begin{aligned}
& U_{i}(x)=\frac{\left|\begin{array}{cccc}
F_{1}(x) & F_{2}(x) & \cdots & F_{i}(x) \\
\left(F_{1}, G_{1}\right) & \left(F_{2}, G_{1}\right) & \cdots & \left(F_{i}, G_{1}\right) \\
\cdot \cdot \cdot \cdot \cdot & \cdot \cdot \cdot \cdot & \cdots & \cdot \\
\left(F_{1}, G_{i-1}\right) & \left(F_{2}, G_{i-1}\right) & \cdots & \left(F_{i}, G_{i-1}\right)
\end{array}\right|}{\sqrt{K_{i-1} K_{i}}}, \\
& V_{i}(x)=\frac{\left|\begin{array}{cccc}
G_{1}(x) & G_{2}(x) & \cdots & G_{i}(x) \\
\left(G_{1}, F_{1}\right) & \left(G_{2}, F_{1}\right) & \cdots & \left(G_{i}, F_{1}\right) \\
\cdot \cdot \cdot \cdot \cdot & \cdot \cdot \cdot & \cdots & \cdot \\
\left(G_{1}, F_{i-1}\right) & \left(G_{2}, F_{i-1}\right) & \cdots & \left(G_{i}, F_{i-1}\right)
\end{array}\right|}{\sqrt{K_{i-1} K_{i}}},
\end{aligned}
$$

$$
K_{i}=\left|\begin{array}{cccc}
\left(F_{1}, G_{1}\right) & \left(F_{2}, G_{1}\right) & \cdots & \left(F_{i}, G_{1}\right) \\
\left(F_{1}, G_{2}\right) & \left(F_{2}, G_{2}\right) & \cdots & \left(F_{i}, G_{2}\right) \\
\cdot \cdot \cdot \cdot \cdot \cdot \cdot & \cdot & \cdot & \cdot \\
\left(F_{1}, G_{i}\right) & \left(F_{2}, G_{i}\right) & \cdots & \left(F_{i}, G_{i}\right)
\end{array}\right| .
$$

That the matrix $V$ is limited, follows from the substitution of

$$
F(x)=H(x)-\sum_{j=1}^{n} U_{j}(x)\left(V_{j}, H\right),
$$

where $H(x)$ is any limited linear form, in $(F, T F) \geqq 0$. We obtain

$$
\sum_{k=1}^{n}\left(V_{k}, H\right)^{2} \leqq(H, T H) \text {. }
$$

Since $T$ is limited it follows from $\mathrm{B} \S 2$ that $V$ is limited. It can be seen directly from the construction that $V_{i}(x)=T U_{i}(x)$, and hence $\left(V_{i}, \bar{F}\right)=0$. To prove the last part of the theorem, let $V^{\prime} V-T=R$. Then $R U_{i}(x)=0$, and also $R \bar{F}_{i}(x)=0$. Since $\left\{u_{i}(x)\right\}$ and $\left\{\bar{F}_{i}(x)\right\}$ form a complete system if $\left\{F_{i}(x)\right\}$ and $\left\{\bar{F}_{i}(x)\right\}$ do, $R=0$ and $V^{\prime} V=T$.

The limited linear forms $\left\{U_{i}(x)\right\}$ and $\left\{V_{i}(x)\right\}$, and the limited linear differential forms $\left\{d Q^{(a)}(\lambda ; x)\right\}$ and $\left\{d R^{(a)}(\lambda ; x)\right\}$ form a biorthogonal system with the basis functions $\left\{r_{0}^{(a)}\right\}$ for an interval $(a, b)$ if the functions $\left\{r_{0}^{(a)}\right\}$ are continuous, monotonic non-decreasing in $(a, b)$; if 


$$
\left(U_{i}, V_{k}\right)=e_{i k}
$$

if for any two intervals $\Delta_{1}$ and $\Delta_{2}$ of $(a, b)$

and if for any interval $\Delta$ of $(a, b)$

$$
\left(\Delta_{1} Q^{(a)}, \Delta_{2} R^{(\beta)}\right)=\left\{\begin{array}{cc}
\Delta_{12} r_{0}^{(a)} & \alpha=\beta, \\
0 & \alpha \neq \beta ;
\end{array}\right.
$$

$$
\left(\Delta Q^{(a)}, V_{i}\right)=\left(\Delta R^{(a)}, U_{i}\right)=0 .
$$

TheOREM II. If for the biorthogonal system $\left(U_{i}(x), V_{i}(x), d Q^{(a)}(\lambda ; x)\right.$, $\left.d R^{(a)}(\lambda ; x)\right)$ with basis functions $\left\{r_{0}^{(a)}\right\}$ for an interval $(a, b)$, the matrix $V$ is limited, and the matrix $\left\{d R^{(a)}\right\}$ is limited with respect to $\left\{r_{0}^{(a)}\right\}$ for the interval $(a, b)$, there exists a positive, symmetric, and limited matrix $T$ such that

$$
V_{i}(x)=T U_{i}(x), \quad d R^{(a)}(\lambda ; x)=T d Q^{(a)}(\lambda ; x) .
$$

Such a matrix $T$ is given by

$$
T=V^{\prime} V+R^{\prime} R
$$

By D and G $\S 2$ it is limited; it is obviously positive and symmetric.

Theorem III. If $T$ is a positive, symmetric, and limited matrix, there exists a biorthogonal system $\left(U_{i}(x), V_{i}(x), d Q^{(a)}(\lambda ; x), d R^{(a)}(\lambda ; x)\right)$ with basis functions $\left\{r_{0}^{(a)}\right\}$ for an interval $(a, b)$, such that the matrix $V$ is limited, the matrix $\left\{d R^{(a)}\right\}$ is limited with respect to the functions $\left\{r_{0}^{(a)}\right\}$ for the interval $(a, b)$, and.

$V_{i}(x)=T U_{i}(x), \quad d R^{(a)}(\lambda ; x)=T d Q^{(a)}(\lambda ; x), \quad T=V^{\prime} V+R^{\prime} R$.

Let $\left\{L_{i}(x)\right\}$ be the characteristic linear forms associated with the matrix $T$ and corresponding to the characteristic numbers $\lambda_{i}$, and $\left\{d P^{(a)}(\lambda ; x)\right\}$ the characteristic linear differential forms for the continuous spectrum $(a, b)$, which form an orthogonal system with basis functions $\left\{p_{0}^{(\alpha)}\right\}$ for $(a, b)$ (I $\$ 2$ ).

A biorthogonal system satisfying the conditions in the theorem is constructed as follows

$$
\begin{gathered}
U_{i}(x)=\frac{L_{i}(x)}{\sqrt{\lambda_{i}}}, \quad V_{i}(x)=\sqrt{\lambda_{i}} L_{i}(x), \quad Q^{(a)}(\lambda ; x)=P^{(a)}(\lambda ; x) ; \\
R^{(a)}(\lambda ; x)=\int_{a}^{\lambda} \lambda d P^{(a)}(\lambda ; x), \quad r_{0}^{(a)}(\lambda)=\int_{a}^{\lambda} \lambda d p_{0}^{(a)}(\lambda) .
\end{gathered}
$$

The spectrum is positive $(J \S 2)$, and hence $U_{i}(x)$ and $V_{i}(x)$ are real. The matrix $V$ is limited, since $L$ is limited (E $\S 2$ ) and the characteristic numbers are finite (I $\S 2)$. The functions $r_{0}^{(a)}$ are continuous, monotonic non-decreasing functions of $\lambda$ in $(a, b)$. If $a \neq 0$, and $\left\{d F^{(a)}\right\}$ is limited 
with respect to $\left\{p_{0}^{(a)}\right\}$, it is limited with respect to $\left\{r_{0}^{(a)}\right\}$. The matrix $\left\{d R^{(a)}\right\}$ is limited with respect to $\left\{r_{0}^{(a)}\right\}$ in any case, for, from the inequality

if follows that

$$
\left(\sum_{j=1}^{n} \lambda_{j} \delta_{j} P^{(a)}\right)^{2} \leqq \sum_{j=1}^{n} \lambda_{j} \frac{\left(\delta_{j} P^{(a)}\right)^{2}}{\delta_{j} p_{0}^{(a)}} \sum_{j=1}^{n} \lambda_{j} \delta_{j} p_{0}^{(a)}
$$

and

$$
\left(\int_{\Delta} \lambda d P^{(a)}\right)^{2} \leqq \int_{\Delta} \lambda \cdot \frac{\left(d P^{(a)}\right)^{2}}{d p_{0}^{(a)}} \int_{\Delta} \lambda d p_{0}^{(a)},
$$

$$
\int_{a}^{b} \frac{\left(d R^{(a)}\right)^{2}}{d r_{0}^{(a)}} \leqq \int_{a}^{b} \lambda \frac{\left(d P^{(a)}\right)^{2}}{d p_{0}^{(a)}}
$$

From $\int_{\Delta_{i}} \lambda d f=\lambda_{i} \Delta_{i} f+\Delta_{i} \lambda\left(f\left(\lambda_{i+1}\right)-f(\bar{\lambda})\right)$ where $\lambda_{i} \leqq \bar{\lambda} \leqq \lambda_{i+1}$, and from $\int_{\Delta_{i}} \lambda d p_{0}^{(a)}=\lambda_{i}^{*} \Delta_{i} p_{0}^{(a)}$, we obtain for $a \neq 0$ and for $\left\{f^{(a)}\right\}$ integrable $\mathrm{H}\left(p_{0}^{(a)}\right)$

$$
\int_{a}^{b} \frac{d r_{i}^{(a)} d f^{(a)}}{d r_{0}^{(a)}}=\int_{a}^{b} \frac{d p_{i}^{(a)} d f^{(a)}}{d p_{0}^{(a)}}
$$

For the case $a=0$ and $b>\epsilon>0$, we define

$$
\int_{0}^{b} \frac{d r_{i}^{(a)} d f^{(a)}}{d r_{0}^{(a)}}=\lim _{\epsilon=0} \int_{e}^{b} \frac{d r_{i}^{(a)} d f^{(a)}}{d r_{0}^{(a)}}=\int_{0}^{b} \frac{d p_{i}^{(a)} d f^{a)}}{d p_{0}^{(a)}}
$$

We have also

$$
\int_{a}^{b} \frac{\left(d R^{(a)}\right)^{2}}{d r_{0}^{(a)}}=\int_{a}^{b} \lambda \frac{\left(d P^{(a)}\right)^{2}}{d p_{0}^{(a)}}
$$

The remainder of the theorem follows from (5), (6), and (7).

If the matrix $T$ is positive definite, the system $\left\{U_{i}(x), d Q^{(a)}(\lambda ; x)\right\}$ is complete.

THEOREM IV. If $\left(U_{i}(x), V_{i}(x), d Q^{(a)}(\lambda ; x), d R^{(a)}(\lambda ; x)\right)$ is a biorthogonal system with basis functions $\left\{r_{0}^{(a)}\right\}$ for an interval $(a, b)$, such that the matrix $V$ is limited, the matrix $\left\{d R^{(a)}\right\}$ limited with respect to $\left\{r_{0}^{(a)}\right\}$ on $(a, b)$, and the system $\left(U_{i}(x), d Q^{(a)}(\lambda ; x)\right)$ complete, then for any sequence $\left\{x_{i}\right\}$ of finite norm, and for any sequence $\left\{y_{i}\right\}$ such that $\left\{U_{i}(y)\right\}$ is of finite norm and $\left\{Q^{(a)}(\lambda ; y)\right\}$ is integrable $H\left(r_{0}^{(a)}\right)$ on $(a, b)$,

$$
\sum_{i} x_{i} y_{i}=\sum_{i} V_{i}(x) U_{i}(y)+\sum_{a} \int_{a}^{b} \frac{d R^{(a)}(\lambda ; x) d Q^{(a)}(\lambda ; y)}{d r_{0}^{(a)}(\lambda)}
$$

Call the difference between the two sides of (11) $F(x)$, and set $x_{i}$ equal to $U_{i j}$ and also to $\Delta q_{i}^{(a)}$. Then by $\mathrm{C} \S 2$ and $(2),\left(F, U_{i}\right)=0=\left(F, \Delta Q^{(a)}\right)$ and therefore $F(x) \equiv 0$.

The relation (11) may hold for other values of $\left\{y_{i}\right\}$ than those stated in 
the theorem; for example, for the biorthogonal system in Theorem III the relation (11) is true for all sequences $\left\{y_{i}\right\}$ of finite norm, by virtue of (8) and (10).

If the columns of the matrix $U$ are of finite norm, and if the system of functions $\left\{q_{i}^{(\alpha)}(\lambda)\right\}$ is integrable $H\left(r_{0}^{(\alpha)}\right)$ on $(a, b)$, we may take $y_{i}=e_{i k}$ and (11) becomes

$$
V^{\prime} U+R^{\prime} Q=E
$$

This relation is true for an orthog nal system which is complete, also for the biorthogonal system (8). If the system consists only of linear forms, (11') expresses that $U$ is the front reciprocal of $V^{\prime}$. Toeplitz* proved (11') for the special case of two matrices which are both limited. That the relation $\left(11^{\prime}\right)$ is not true for every biorthogonal system of limited linear forms is shown by the following example

$$
U_{i}(x)=L_{i+1}(x)-L_{1}(x), \quad V_{i}(x)=L_{i+1}(x),
$$

where $\left\{L_{i}(x)\right\}$ is a complete orthogonal system of linear forms.

Theorem V. Let $T$ be a positive definite, symmetric, limited matrix; let $\left(\bar{U}_{i}(x), \bar{V}_{i}(x)\right)$ form a biorthogonal system of limited linear forms with the matrix $\bar{V}$ limited, the system $\left\{\bar{U}_{i}(x)\right\}$ complete, and $\bar{V}_{i}(x)=T \bar{U}_{i}(x)$; let $\left(U_{i}(x), V_{i}(x), d Q^{(a)}(\lambda ; x), d R^{(a)}(\lambda ; x)\right.$ ) form a biorthogonal system of limited linear forms and linear differential forms with the basis functions $\left\{r_{0}^{(a)}\right\}$ for the interval $(a, b), V$ limited, $\left\{d R^{(a)}\right\}$ limited with respect to $\left\{r_{0}^{(a)}\right\}$ on $(a, b), V_{i}(x)=T U_{i}(x), d R^{(a)}(\lambda ; x)=T d Q^{(a)}(\lambda ; x)$ and the system of $\left\{U_{i}(x)\right\}$ and $\left\{d Q^{(a)}(\lambda ; x)\right\}$ complete, then $\sum_{j} \bar{u}_{i j} v_{k j}=\sum_{j} \bar{v}_{i j} u_{k j}$ and $\sum_{j} \bar{u}_{i j} r_{j}^{(a)}(\lambda)=\sum_{j} \bar{v}_{i j} q_{j}^{(a)}(\lambda)$ are the coefficients of a complete orthogonal system with the basis functions $\left\{r_{0}^{(a)}\right\}$ for the interval $(a, b)$.

The orthogonal property follows from Theorem 4, since

$$
\begin{aligned}
& \sum_{j}\left(U, \bar{V}_{j}\right)\left(\bar{V}_{j}, U_{k}\right)=\sum_{j}\left(U_{i}, \bar{V}_{j}\right)\left(\bar{U}_{j}, V_{k}\right)=\left(U_{i}, V_{k}\right)=e_{i k}, \\
& \sum_{j}\left(\Delta_{1} Q^{a)}, \bar{V}_{j}\right)\left(\bar{V}_{j}, \Delta_{2} Q^{(\beta)}\right)=\sum_{j}\left(\Delta_{1} Q^{(a)}, \bar{V}_{j}\right)\left(\bar{U}_{j}, \Delta_{2} R^{(\beta)}\right) \\
& =\left(\Delta_{1} Q^{(a)}, \Delta_{2} R^{(\beta)}\right)=\left\{\begin{array}{cc}
\Delta_{12} r_{0}^{(a)} & \alpha=\beta, \\
0 & \alpha \neq \beta,
\end{array}\right. \\
& \sum_{j}\left(U_{i}, \bar{V}_{j}\right)\left(\bar{V}_{j}, \Delta Q^{(a)}\right)=\sum_{j}\left(U_{i}, \bar{V}_{j}\right)\left(\bar{U}_{j}, \Delta R^{(a)}\right)=\left(U_{i}, \Delta R^{(a)}\right)=0 .
\end{aligned}
$$

The system is complete, for if $C(x)$ were orthogonal to $\bar{V} U_{i}(x)$ and $\bar{V} Q^{(a)}(\lambda ; x)$, then $\bar{V}^{\prime} C(x)$ would be orthogonal to $U_{i}(x)$ and $d Q^{(a)}(\lambda ; x)$.

* Die Jacobische Transformation der quadratischen Formen von unendlichvielen Veränderlichen, $\mathrm{Nachrichten} \mathrm{der} \mathrm{Kgl.} \mathrm{Gesellschaft} \mathrm{der} \mathrm{Wissenschaften} \mathrm{zu}$ G öt t i n g e n, Math-Phys. Kl., 1907, p. 101. 
4. LINEAR EQUATIONS WITH UNSYMMETRIC SYSTEMS OF COEFFICIENTS

Theorem VI. If, for the limited matrix $A$, there exist for real values of $\lambda$ real characteristic linear forms and linear differential forms of the equations (5) and (6) which form a complete system together with limited linear forms $F_{i}(x)$ such that $A F_{i}(x)=0$, then there exists a positive definite, symmetric, and limited matrix $T$ such that $A T$ is symmetric.

Let $\left\{V_{i}(x)\right\}$ be the characteristic linear forms for which the matrix $V$ may be assumed to be limited, and $\left\{d R^{(a)}(\lambda ; x)\right\}$ the system of characteristic linear differential forms for which $\left\{d R^{(a)}\right\}$ is limited with respect to $\left\{r_{0}^{(a)}\right\}$ for the interval $(a, b)$. It is obvious that the matrix $F$ may be assumed to be limited. The matrix $T$

$$
T=V^{\prime} V+F^{\prime} F+R^{\prime} R
$$

is positive definite, symmetric, and limited, and the matrix $A T$ is symmetric.

Theorem VII. If $A$ is a limited matrix, not identically zero, and if there exists a positive definite, symmetric, limited matrix $T$ such that AT is symmetric, then there exist real characteristic linear forms and linear differential forms of the equations (5) and (6), which form a complete system with limited linear forms $F_{i}(x)$ such that $A F_{i}(x)=0$.

Corresponding to the matrix $T$ there exists by Theorem I a biorthogonal system of limited linear forms $\left\{\bar{U}_{i}(x)\right\}$ and $\left\{\bar{V}_{i}(x)\right.$, with the matrix $\bar{V}$ limited, $\left\{\bar{U}_{i}(x)\right\}$ complete, and $\bar{V}_{i}(x)=T \bar{U}_{i}(x)$. The matrix

$$
\bar{B}=\bar{U} A \bar{V}^{\prime}
$$

exists and is symmetric. To show that it is limited we introduce the biorthogonal system $\left(U_{i}(x), V_{i}(x), d Q^{(a)}(\lambda ; x), d R^{(a)}(\lambda ; x)\right)$ defined by (8). Let $\bar{l}_{i k}=\sum_{j} \bar{u}_{k j} v_{i j}$ and $\bar{p}_{i}^{(a)}(\lambda)=\sum_{j} \bar{u}_{i j} r_{j}^{(a)}(\lambda)$, then by $\mathrm{E}$ and $\mathrm{H} \S 2$ and Theorem $\mathrm{V}$ the matrix $\bar{L}$ is limited and $\left\{d \bar{P}^{(a)}\right\}$ is limited with respect to $\left\{r_{0}^{(a)}\right\}$ on $(a, b)$. Applying relation (11) we obtain

$$
\bar{U}=U^{\prime} \bar{L}+Q^{\prime} \bar{P}, \quad \bar{V}=V^{\prime} \bar{L}+R^{\prime} \bar{P} .
$$

Substitute these expressions in the elements of $\bar{B}$, and, since the summation signs may be interchanged on account of the form of $\bar{U}$ and $\bar{V}$, we obtain

$$
\bar{B}=\bar{U} A \bar{V}^{\prime}=\bar{L}^{\prime}\left(U A V^{\prime} \bar{L}\right)+\bar{L}\left(U A R^{\prime} \bar{P}\right)+\bar{P}^{\prime} Q A V^{\prime} \bar{L}+\bar{P}^{\prime} Q A R^{\prime} \bar{P} .
$$

It is easy to see that $B$ is limited if zero is not a limiting point of the point spectrum and does not belong to the continuous spectrum, for then the matrix $U$ is limited, and the matrix $\left\{d Q^{(a)}\right\}$ is limited with respect to $\left\{r_{0}^{(a)}\right\}$.

The first term of $\bar{B}$ is a limited matrix if the matrix $B=U A V^{\prime}$ is limited. The following argument shows that $B$ is limited in any case. The maximum 
value of $\left|\sum_{i, k=1}^{i, k=n} b_{i k} x_{i} x_{k}\right|$ for $\sum_{i=1}^{i=n} x_{i}^{2} \leqq 1$, is equal to the absolute value of that characteristic number which is largest in absolute value and satisfies

$$
\lambda^{(n)} z_{i}=\sum_{k=1}^{n}\left(\frac{1}{\sqrt{\lambda_{i}}} \sum_{j, l} l_{i j} a_{j l} l_{k l} \sqrt{\lambda_{k}}\right) z_{k} \quad(i=1,2, \cdots, n) .
$$

We may assume that $\sum_{i=1}^{i=n} \lambda_{i} z_{i}^{2}=1$, and hence

$$
\lambda^{(n)}=\sum_{i, k=1}^{n}\left(\sum_{j, l} l_{i j} a_{j l} l_{k l}\right) \sqrt{\lambda_{i}} z_{i} \sqrt{\lambda_{k}} z_{k} .
$$

Since the matrix $L A L^{\prime}$ is limited (D and $\mathrm{E} \S 2$ ), $\left|\lambda^{(n)}\right| \leqq M$ for all values of $n$, and the matrix $B$ is limited.

If $C$ denotes the second term, $C^{\prime}$ denotes the third term. If zero is not a limiting point of the point spectrum, $C$ is obviously limited, and hence also $C^{\prime}$. If zero does not belong to the continuous spectrum, $C^{\prime}$ is limited, and hence $C$ is also limited. To consider the remaining case, let

$$
C^{(\epsilon)}=\bar{L}^{\prime}\left(U A R^{\prime} \bar{P}_{\epsilon}^{b}\right) \text {, }
$$

where $b>\epsilon>0$, and construct $C^{(\epsilon)} C^{(\epsilon) \prime}$, which may be expressed as follows

$$
C^{(e)} C^{(e) \prime}=\bar{L}^{\prime} U A R^{\prime} \bar{P}_{\epsilon}^{b} \bar{P}^{\prime} Q_{\varepsilon}^{b} A V^{\prime} \bar{L} .
$$

From the orthogonal property (4) of $\left\{d \bar{P}^{(a)}(\lambda ; x)\right\}$, and results obtained in proving the first term limited, it follows that

$$
\left|C^{(\epsilon)} C^{(e) \prime}(x, x)\right| \leqq M^{2},
$$

and since $\lim _{\boldsymbol{\epsilon}=0} C_{i k}^{(\epsilon)}=C_{i k}$, the matrices $C$ and $C^{\prime}$ are limited $(F \S 2)$ in all cases.

The fourth term of $\bar{b}_{i k}$ is limited if zero does not belong to the continuous spectrum. In case it does, let $\epsilon$ and $\bar{\epsilon}$ be positive and less than $b$; divide $(\epsilon, b)$ into $n$ subintervals $\Delta_{i}$ and $(\bar{\epsilon}, b)$ into $n$ subintervals $\bar{\Delta}_{i}$. Let

$$
\begin{aligned}
& d_{i, k}^{(n, \bar{n})}=\sum_{a, j} \sum_{m=1}^{n} \frac{\Delta_{m}}{p_{i}^{(a)} \Delta_{m} q_{j}^{(a)}} \sum_{m} r_{0}^{(a)} a_{l, \beta} \sum_{j l}^{\bar{n}} \frac{\Delta_{\bar{m}} r_{l}^{(\beta)} \Delta_{m} \bar{p}_{k}^{(\beta)}}{\Delta_{\bar{m}} r_{0}^{(\beta)}} \\
&=\sum_{a, j} \sum_{m=1}^{n} \frac{\Delta_{m} \bar{p}_{i}^{(a)} \Delta_{m} r_{j}^{(a)}}{\Delta_{m} r_{0}^{(a)}} \sum_{l, \beta} a_{j l} \sum_{\bar{m}=1}^{\bar{n}} \frac{\Delta_{\bar{m}} q_{l}^{(\beta)} \Delta_{\bar{m}} \bar{p}_{k}^{(\beta)}}{\Delta_{\bar{m}} r_{0}^{(\beta)}},
\end{aligned}
$$

then $D^{(n, \bar{n})}(x, x)$ is completely continuous $(\mathrm{L} \S 2)$ for each value of $n$ and $\bar{n}$, since it is composed of a limited form $A(x, x)$ and completely continuous forms (L $\S 2)$; but $D^{(n, \bar{n})}$ is not symmetric for $n$ and $\bar{n}$ are not equal. Form

$$
\sum_{j} d_{i j}^{(n, \bar{n})} d_{k j}^{(n, \bar{n})}=\sum_{a, j} \sum_{m=1}^{n} \frac{\Delta_{m} \bar{p}_{i}^{(\alpha)} \Delta_{m} q_{j}^{(\alpha)}}{\Delta_{m} r_{0}^{(\alpha)}} \sum_{l, \beta} \bar{a}_{j l} \sum_{\bar{m}=1}^{n} \frac{\Delta_{\bar{m}} r_{l}^{(\beta)} \Delta_{\bar{m}} \bar{p}_{k}^{(\beta)}}{\Delta_{\bar{m}} r_{0}^{(\beta)}},
$$


where $|\bar{A}(x, x)| \leqq M^{2} \sum_{i} x_{i}^{2}$ and $M$ is independent of $\bar{n}$. The maximum value of $\left|D^{(n, \bar{n})} D^{(n, \bar{n})^{\prime}}(x, x)\right|$ for $\sum_{i} x_{i}^{2}=1$, since it is a quadratic form, is equal to the absolute value of that characteristic number $\mu$, which is largest in absolute value, for the equations

$$
\mu z_{i}=\sum_{a, j} \sum_{m=1}^{n} \frac{\Delta_{m} \bar{p}_{i}^{(a)}}{\sqrt{\Delta_{m} r_{0}^{(\alpha)}}} \cdot \frac{1}{\sqrt{\lambda_{m}^{(a)}}} \frac{\Delta_{m} q_{j}^{(\alpha)}}{\sqrt{\Delta_{m} p_{0}^{(\alpha)}}} \sum_{l, \beta} \bar{a}_{j l} \sum_{\bar{m}=1}^{n} \frac{\Delta_{\bar{m}} r_{l}^{(\beta)}}{\lambda_{\bar{m}}^{(\beta)} \sqrt{\Delta_{\bar{m}} p_{0}^{(\beta)}}} \cdot \sqrt{\lambda_{\bar{m}}^{\beta}} \frac{\Delta_{\bar{m}} \bar{p}_{k}^{(\beta)}}{\sqrt{\Delta_{\bar{m}} r_{0}^{(\beta)}}} z_{k},
$$

where $\left\{z_{i}\right\}$ is of finite norm and $\lambda_{n}^{(a)} \Delta_{m} p_{0}^{(a)}=\Delta_{m} r_{0}^{(a)}$. Multiply both sides by $\sqrt{\lambda_{m}^{(a)}} \Delta_{m} p_{i}^{(a)} / \sqrt{\Delta_{m}} r_{0}^{(a)}$ and sum with respect to $i$. Then multiply both sides by $\sum_{i} \sqrt{\lambda_{m}^{(a)}} \Delta_{m} p_{i}^{(a)} z_{i} / \sqrt{\Delta_{m} r_{0}^{(a)}}$ and sum with respect to $m$ and $\alpha$. We may assume that

and since

$$
\sum_{a, m}\left(\sum_{i} \frac{\sqrt{\lambda_{m}^{(a)} \Delta_{m} p_{i}^{(a)}}}{\sqrt{\Delta_{m} r_{0}^{(a)}}} z_{i}\right)^{2}=1,
$$

$$
\left|\sum_{\cdot,, j, m} x_{m}^{(\alpha)} \frac{\Delta_{m} q_{j}^{(\alpha)}}{\sqrt{\Delta_{m} p_{0}^{(\alpha)}}} \sum_{l, \beta} \bar{a}_{j l} \sum_{\bar{m}} \frac{\Delta_{\bar{m}} r_{l}^{(\beta)}}{\lambda_{\bar{m}}^{(\beta)} \sqrt{\Delta_{\bar{m}} p_{0}^{(\beta)}}} x_{\bar{m}}^{(\beta)}\right| \leqq M^{2} \sum_{a} \sum_{m}\left(x_{m}^{(\alpha)}\right)^{2},
$$

it follows that $|\mu| \leqq M^{2}$ for all values of $n$ and $\bar{n}$, and hence

and also

$$
\left|D^{(n, \bar{n})}(x, x)\right| \leqq M \sum_{i} x_{i}^{2},
$$

$$
\left|\bar{P}^{\prime} Q_{e}^{b} A R^{\prime} \bar{P}_{e}^{b}(x, x)\right| \leqq M \sum_{i} x_{i}^{2},
$$

and in consequence of ( $\mathrm{F} \S 2)$ the last term of $\bar{b}_{i k}$ is limited.

Since $\bar{B}$ is limited and symmetric, there exist (I $\S 2$ ) characteristic linear forms $\left\{\bar{L}_{i}(x)\right\}$ corresponding to real characteristic numbers $\bar{\lambda}_{i}$ of the equations

$$
\overline{\bar{\lambda}}_{i} \overline{\bar{l}}_{i k}=\sum_{j} \bar{b}_{k j} \overline{\bar{l}}_{i j}
$$

and characteristic linear differential forms $\left\{d \overline{\bar{P}}^{(\beta)}(\lambda ; x)\right\}$ limited with respect to $\left\{\bar{p}_{0}^{(\beta)}\right\}$ for an interval $(\overline{\bar{a}}, \bar{b})$, of the equations

$$
\int_{\Delta} \lambda d \overline{\bar{p}}_{k}^{(\boldsymbol{\beta})}=\sum_{j} \bar{b}_{k j} \Delta \bar{p}_{j}^{(\beta)},
$$

where $\Delta$ is any subinterval $\Delta$ of $(\overline{\bar{a}}, \overline{\bar{b}})$. Let

$$
\overline{\bar{v}}_{i k}=\sum_{j} \overline{\bar{l}}_{i j} \bar{v}_{j k}, \quad \overline{\bar{r}}_{k}^{(\beta)}(\lambda)=\sum_{j} \overline{\bar{p}}_{j}^{(\beta)}(\lambda) \bar{v}_{j k} .
$$

Then $\left\{\overline{\bar{V}}_{i}(x)\right\}$ are characteristic linear forms, corresponding to $\left\{\bar{\lambda}_{i}\right\}$, of the equations

$$
\overline{\bar{\lambda}}_{i} \overline{\bar{v}}_{i k}=\sum_{j} a_{k j} \overline{\bar{v}}_{i j}
$$


and $\left\{d \overline{\bar{R}}^{(\beta)}(\lambda ; x)\right\}$ are characteristic linear differential forms, for the continuous spectrum $(\overline{\bar{a}}, \overline{\bar{b}})$, of the equations

$$
\int_{\Delta} \lambda d \overline{\bar{r}}_{k}^{(\beta)}(\lambda)=\sum_{j} a_{k j} \Delta \overline{\bar{r}}_{j}^{(\beta)}
$$

where $\Delta$ is any subinterval of $(\overline{\bar{a}}, \overline{\bar{b}})$.

Apply (7) and (11) to $B\left(x^{*}, z^{*}\right)$, where $x_{i}{ }^{*}=\bar{V}_{i}(x), z_{i}{ }^{*}=\bar{V}_{i}(z)$ and we obtain

$$
A(x, y)=\sum_{j} \overline{\bar{\lambda}}_{j} \overline{\bar{V}}_{j}(x) \overline{\bar{V}}_{j}(z)+\sum_{\beta} \int_{\overline{\bar{a}}}^{\bar{b}} \lambda \cdot \frac{d \overline{\bar{R}}^{(\beta)}(\lambda ; x) d \overline{\bar{R}}^{(\beta)}(\lambda ; z)}{d \overline{\bar{p}}_{0}^{(\beta)}(\bar{\lambda})},
$$

for all sequences $\left\{x_{i}\right\}$ of finite norm, and all sequences $\left\{y_{i}\right\}$ such that $y_{i}=\sum_{k} t_{i k} z_{k}$ where $\left\{z_{i}\right\}$ is of finite norm. From this development (16) it follows that any limited linear form $F(x)$ which is orthogonal to $\left\{\bar{V}_{i}(x)\right\}$ and $\left\{d \bar{R}^{(\beta)}(\lambda ; x)\right\}$ is such that $A F(x)=0$, and the theorem is proved.

If the adjoint system of equations

$$
\bar{\lambda}_{i} \overline{\bar{u}}_{i k}=\sum_{j} a_{j k} \overline{\bar{u}}_{i j}, \quad \int_{\Delta} \lambda d \overline{\bar{q}}_{k}(\lambda)=\sum_{j} a_{j k} \Delta \overline{\bar{q}}_{j}
$$

where $\Delta$ is any subinterval of $(\overline{\bar{a}}, \overline{\bar{b}})$, have characteristic forms $\left\{\overline{\bar{U}}_{i}(x)\right\}$ and $\left\{d \overline{\bar{Q}}^{(\beta)}(\lambda ; x)\right\}$, then for the same values of $\lambda$ the equations (12) and (13) have solutions, and $\left\{T \overline{\bar{U}}_{i}(x)\right\}$ and $\left\{T d \overline{\bar{Q}}^{(\beta)}(\lambda ; x)\right\}$ satisfy (14) and (15).

If

$$
\overline{\bar{u}}_{i k}=\sum_{j} \overline{\bar{l}}_{i j} \bar{u}_{j k}, \quad \overline{\bar{q}}_{k}^{(\beta)}(\lambda)=\sum_{j} \overline{\bar{p}}_{j}^{(\beta)}(\lambda) \bar{u}_{j k}
$$

exist and are the coefficients of limited systems, the forms $\left\{\bar{U}_{i}(x)\right\}$ and $\left\{d \bar{Q}^{(\beta)}(\lambda ; x)\right\}$ are characteristic forms of (17) corresponding to $\left\{\bar{\lambda}_{i}\right\}$ and $(\bar{a}, \bar{b})$, form a biorthogonal system with $\left\{\overline{\bar{V}}_{i}(x)\right\}$ and $\left\{d \overline{\bar{R}}^{(\beta)}(\lambda ; x)\right\}$, and the development (16) becomes

$$
A(x, y)=\sum_{j} \bar{\lambda}_{j} \bar{V}_{j}(x) \bar{U}_{j}(y)+\sum_{\beta} \int_{\bar{a}}^{\bar{b}} \lambda \cdot \frac{d \bar{R}^{(\beta)}(\lambda ; x) d \bar{Q}^{(\beta)}(\lambda ; y)}{d \bar{p}_{0}^{(\beta)}},
$$

where $\left\{x_{i}\right\}$ and $\left\{z_{i}\right\}$ are any sequences of finite norm, and $y_{i}=\sum_{k} t_{i k} z_{k}$.

Some sufficient conditions that (17) have characteristic forms are: 1), that the matrix $U$ be limited, which is so in case the values of $\lambda$ belonging to the spectrum are $>M>0 ; 2$ ), that $A=T K$ where $K$ is a symmetric limited matrix; 3$)$, that $A(x, x)$ be a completely continuous function $(\mathrm{K} \S 2)$.

The following example shows that for a limited matrix $A$ such that $A T$ is 
symmetric, where $T$ is a positive definite, symmetric, and limited matrix, the equations (14) and (15) may have characteristic forms while the adjoint system (17) does not. Let

$$
u_{i k}=\left\{\begin{array}{rl}
\lambda_{i} & i=k \\
-\lambda_{i} & i=k-1 \\
0 & i \neq k, i \neq k-1,
\end{array}\right.
$$

where $\lambda_{i}=2-1 / 2^{i-1}$. The only solutions of (17) correspond to $\lambda_{i}$, but they are not of finite norm. The equations (14) have solutions of finite norm for $\lambda=\lambda_{i}$ and they form a complete system. In fact, these equations (14) have solutions of finite norm for $|\lambda|<2$. If $\lambda_{i}=1$, the equations (17) have no solutions whatever, and the equations (14) have solutions of finite norm for $|1-\lambda|<1$. The solutions are $x_{i}=(1-\lambda)^{i-1}$.

Theorems I and II enable us to give the following form to the Theorems VI and VII.

Theorem VIII. The necessary and sufficient condition that for a limited matrix $A$, there exist real characteristic forms and characteristic linear differential forms of the equations (5) and (6), such that they form a complete system with limited linear forms $F(x)$ such that $A F_{i}(x) \equiv 0$, is the existence of two matrices $U$ and $V$ such that $U$ is the rear reciprocal of $V^{\prime}$, the matrix $U A V^{\prime}$ is symmetric, the rows of $U$ are of finite norm, $V$ is a limited matrix, and the systems $\left\{U_{i}(x)\right\}$ and $\left\{V_{i}(x)\right\}$ are complete.

Bryn Mawr College. 